Article

\title{
Frequency Diversity Gain of a Wideband Radar Signal
}

\author{
Mengmeng Shen ${ }^{1}$, Feng $\mathrm{He}^{1, *}$, Zhen Dong ${ }^{1}$, Xing Chen ${ }^{1}\left[\right.$, Lei $\mathrm{Yu}^{1}{ }^{1}$ and Manqing $\mathrm{Wu}^{2}$ \\ 1 College of Electronic Science and Technology, National University of Defense Technology(NUDT), \\ No. 109 Deya Road, Changsha 410073, China; mengss73@163.com (M.S.); dongzhen@nudt.edu.cn (Z.D.); \\ chenxing13@nudt.edu.cn (X.C.); yulei17@nudt.edu.cn (L.Y.) \\ 2 China Electronics Technology Group Corporation, Beijing 100043, China; mengs73@nudt.edu.cn \\ * Correspondence: hefeng@vip.163.com
}

\section{check for}

updates

Citation: Shen, M.; He, F.; Dong, Z.; Chen, X.; Yu, L.; Wu, M. Frequency Diversity Gain of a Wideband Radar Signal. Remote Sens. 2021, 13, 4885. https://doi.org/10.3390/rs13234885

Academic Editor: Antonio Iodice

Received: 11 October 2021

Accepted: 25 November 2021

Published: 1 December 2021

Publisher's Note: MDPI stays neutral with regard to jurisdictional claims in published maps and institutional affiliations.

Copyright: (c) 2021 by the authors. Licensee MDPI, Basel, Switzerland. This article is an open access article distributed under the terms and conditions of the Creative Commons Attribution (CC BY) license (https:// creativecommons.org/licenses/by/ $4.0 /)$.

\begin{abstract}
Wideband radar has high-range directional resolution, which can effectively reduce the fluctuation of echo and improve the detection probability of a target under the same detection probability requirement. In this paper, a unified wideband radar $\chi^{2}$ distribution target model with more practical significance is innovatively established, on which the probability density function and detection probability function of Swerling 0, Swerling II and Swerling IV targets are analyzed, respectively. A generalized "frequency diversity gain" of wideband radar is proposed and defined based on the contradiction between suppression of fluctuation and accumulation loss, which represents the ratio of Signal-to-Noise Ratio (SNR) gain between broadband signal and reference bandwidth signal under the same condition (when the reference bandwidth is used, the radar target has only one range unit), and the mathematical relation equation of the target detection performance and signal bandwidth (equivalent to the number of distinguishable range elements of the target) is given. A Monte Carlo simulation experiment is designed. Based on the target model established in this paper, the optimal number of target range units corresponding to different detection probability requirements is obtained, which verifies the correctness of the concept proposed in this paper.
\end{abstract}

Keywords: wideband radar; Swerling distribution; incoherent accumulation; target detection; frequency diversity gain

\section{Introduction}

Narrowband radar signal has the advantages of simple processing steps, high SNR and low hardware requirements, so it is often used in traditional target detection. However, with the development of wideband radar and its related technologies, wideband signals are gradually applied to the detection of radar targets [1,2].

Echo accumulation is an important means to improve the performance of weak target detection. It can be divided into coherent accumulation and incoherent accumulation according to the different accumulation methods. For narrowband signals, the range resolution is low, which is usually insufficient to divide the target into multiple extension units. There is no phenomenon of incoherence between the scattering points of the target, which means that the echo of the scattering points of the target is processed by coherent accumulation; that is, it is accumulated before the envelope detection. Wideband signal has a high range resolution, and can usually distinguish the target into multiple range units in the range dimensions. The phenomenon of incoherence occurs between the echoes of each range unit. Generally, only incoherent accumulation can be carried out; that is, accumulation can be carried out after the envelope detection, so the phase information of the echo is lost $[3,4]$.

The target echo of a wideband radar is usually superimposed by the echo of multiple scattering range units of the target, which enables it to have a better suppression ability of target Radar Cross-Section (RCS) fluctuation. Meanwhile, the incoherent accumulation loss of the echo of multiple range units of the target is also an important factor affecting 
the detection performance of a wideband radar [5]. If the scattering characteristics of the target are predicted in advance and matched waveform signals are transmitted or filtered by matched filters, the same detection performance as narrowband signals can be achieved on the basis of high resolution [6,7]. However, most of the target scattering characteristics are unknown and difficult to obtain, so only incoherent accumulation can be carried out. Although incoherent accumulation brings additional SNR loss, it also inhibits the fluctuation loss, and the detection performance of the target is the synthesis of these two factors. Under the influence of the balance of the two factors, it is actually the greatest bandwidth selection process.

Many scholars at home and abroad have also studied the advantages and disadvantages of wideband radar and narrowband radar in terms of target detection performance.

Dai, F. derived the wideband undulating target model, wideband rayleigh target model [8], wideband rice target model and their corresponding narrowband echo model, deduced the radar detection probability under the respective models and, under the premise of appropriate bandwidth, obtained the conclusion that wideband radar has better detection performance than narrowband radar. This conclusion can also be seen in many other similar pieces of literature $[9,10]$.

Xiao, J. et al., proposed that according to the resolution ability of radar signal to strong scattering points on the target [11], signal bandwidth can be divided into narrowband, wideband and critical bandwidth. Let the number of scattering points corresponding to the maximum echo accumulation gain be the number of effective scattering points; when the increase in bandwidth increases the number of effective scattering points of the target, it indicates that the increase in bandwidth is beneficial to the detection of the target. Otherwise, it is not conducive to target detection. However, this paper only gives the analysis method and thinking of optimal bandwidth, and does not give a specific calculation and solution to this problem.

Jia, S. suggested that the target from the point target into extended target with the gradual change from narrowband to wideband signal bandwidth [12], the echo SNR will present an initial decrease after the increase, and the bandwidth corresponding to the highest point of the input signal-to-clutter ratio (SCR) of the radar system is defined as the optimal detection bandwidth. However, the specific theoretical derivation and experimental results are not given in the paper. Li, Y. et al., proposed that the clutter sidelobe width of wideband radar echo has a linear relationship with fractional bandwidth [13], and the SCR will increase significantly with the increase in fractional bandwidth. The specific derivation process and experimental results were given, but the Equation for the relationship between signal-to-clutter ratio and absolute bandwidth was not given yet.

In the traditional sense, frequency diversity means that in the process of signal sending and receiving, multiple transmitters are provided to irradiate the target with a set of narrowband signals of different frequencies to obtain a set of unrelated echo signals. These data can effectively reduce the fluctuation loss of echo, so as to improve the detection performance of the target. Experimental results show that the performance of the traditional frequency diversity is closely related to the detection probability. When the detection probability $\mathrm{P}_{\mathrm{d}}=0.8$, two independent frequencies can provide gain of $2.7 \mathrm{~dB}$, and the gain of three transmitters is $3.6 \mathrm{~dB}$, and that of four transmitters is $4.1 \mathrm{~dB}$, the cumulative gain efficiency decreases as the number of transmitters increases. Considering the economic factors of hardware equipment and the complexity of data processing, generally two transmitters are used at most [14].

Different from narrowband radar, wideband radar can effectively suppress echo fluctuation through incoherent accumulation, offset the reduction in SNR caused by accumulation loss, and improve target detection performance, which can also achieve the effect of "frequency diversity gain". Fran, C. et al., analyzed the ability of wideband radar to eliminate clutter [15]. Through frequency diversity, target fading can be effectively alleviated, thus improving radar's detection ability to target [16]. When detecting a $15 \mathrm{~m}$ air target with a detection probability of $\mathrm{P}_{\mathrm{d}}=0.8$, the target should be resolved into at least 
5-10 range units, and the number of distinguishable range units increases with the increase in detection probability requirements.

The analysis of target detection performance of wideband radar is a traditional and thorny problem. As in the above analysis, many experts and scholars have also carried out research; their research shows that the wideband signal in a high detection probability interval has better detection performance, such as the exact value of the detection probability interval, the degree of the improvement of the detection performance, the optimal bandwidth value, etc. The detailed and unified derivation and conclusion are not given in previous research. Based on the previous work, this paper makes a unified and in-depth analysis of the detection performance of wideband radar, analyzes the role of bandwidth in the process of target detection from multiple angles and tries to give specific and accurate conclusions.

Based on the wideband signal transceiver model and detection theory, the author analyzes the influence of bandwidth on detection results, deduces the relationship between the probability of wideband signal target detection and bandwidth size, gives the definition of wideband radar frequency diversity gain, designs a series of simulation experiments and obtains the optimal signal bandwidth under specific conditions.

\section{Wideband Radar $\chi^{2}$ Distributed Target Model}

For wideband radar, the size of the distributed extended target is much larger than the range dimensional resolution units, so the target can no longer be treated as the ideal point scattering target. In order to further analyze the influence of radar bandwidth on target detection performance, a unified wideband radar $\chi^{2}$ target model is established in this section to study the RCS characteristics of Swerling 0, Swerling II and Swerling IV targets under different bandwidths.

The independent sample of the target RCS measurement can be obtained from the frequency domain, so one measurement sample of target RCS can be obtained from each standard bandwidth. For simple analysis, it is considered that target RCS obtained from different standard bandwidths obey the same distribution. In other words, the measurement sample of target RCS can be obtained on each range unit of the target. For the sake of simplicity of analysis, it is still assumed that the target RCS of each range unit obeys the same distribution. The two ideas are consistent in principle, and both can show the independence between the target echoes of wideband radar. In order to facilitate analysis, the second idea is used for research and modeling in this paper.

In order to give a unified representation of target distribution under wideband radar, a unified target model is established in this section. Assume that the length of the target is $l$ and there are $N$ scattering points that are independent of each other. Assume that the number of scattering points in each range unit of the target is the same, and use $n$ to represent the number:

$$
n=\frac{N}{m}=\frac{N}{\frac{l}{\rho}}=\frac{N}{\frac{2 l B}{c}}=\frac{c N}{2 l B}
$$

where $m$ is the number of target range units, whose value is proportional to signal bandwidth $B ; c$ is the speed of light and $\rho$ is range directional resolution. Figure 1 shows the relationship between signal bandwidth and the number of target range units; $B_{i}$ represents the bandwidth and $\rho_{i}$ is the resolution of range direction corresponding to different bandwidths. With the increase in bandwidth, the range resolution of the signal also increases, and the number of target-distinguishable units increases, but the number of scattered points within each range unit decreases. 


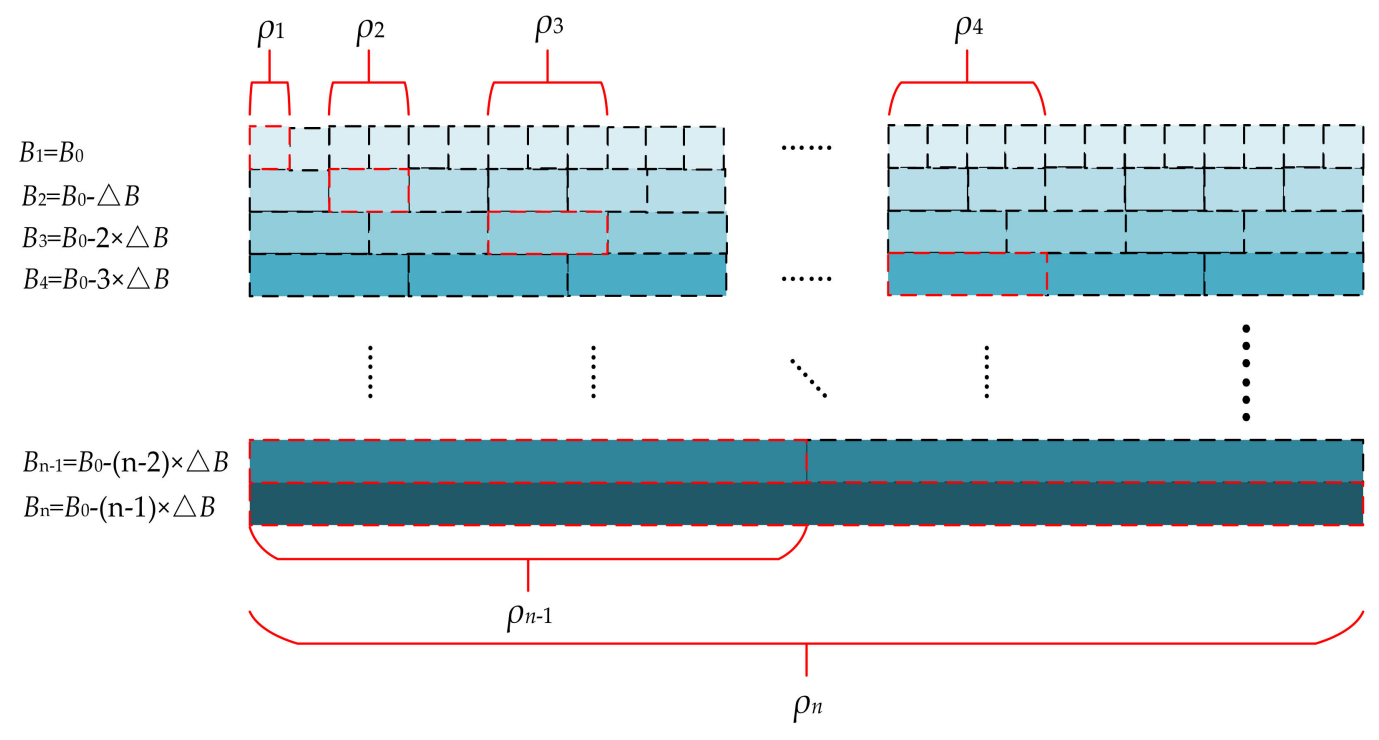

Figure 1. Schematic diagram of wideband target range resolution unit.

In the optimal case, each scattering point of the target is separately distinguished into a range unit by the wideband radar. Theoretically, there will be no fluctuation of the target, and the target RCS can be expressed as:

$$
\sigma=\sum_{i=1}^{m} \sigma_{i}
$$

where $\sigma_{i}$ is the RCS of each scattered point, and the RCS of the target is the scalar sum of all scattered points. In fact, it is impossible for the wideband radar to completely distinguish each scattering point of the target, and because of the existence of random phase, the signal of each range unit of the target cannot achieve the complete coherent accumulation, so it often does not exist in reality. However, the number of targets in each range unit of wideband radar decreases with the increase in bandwidth, and the fluctuation of RCS can be effectively suppressed. More generally, there are multiple scattering points in each range element of the target, so the RCS of the target in a single range element can be expressed as:

$$
\sigma_{i}=\left|\sum_{j=1}^{n_{i}} \sqrt{a_{j}} \exp \left(\frac{4 \pi j R_{x}(j)}{\lambda}\right)\right|^{2}
$$

where $R_{x}(j)$ is the relative position of each scattering point, and Equations (2) and (3) give the RCS of each range unit of the distributed extended target under wideband radar, corresponding to a unfluctuating target and a fluctuating target, respectively. For fluctuating targets, different fluctuating distribution will have certain differences based on Equation (3), and specific analysis and research will be carried out later.

The range units of wideband radar are independent from each other, so their echoes are also irrelevant. Without coherent matching, the target echo is the sum of range units (different from the vector sum between the echoes of scattering points of narrowband radar target), equivalent to the target RCS is the sum of range units, which can be expressed as:

$$
\sigma_{0}=\sum_{i=1}^{m} \sigma_{i}=\sum_{i=1}^{m}\left|\sum_{j=1}^{n} \sqrt{a_{j}} \exp \left(\frac{4 \pi j R_{x}(j)}{\lambda}\right)\right|^{2}
$$

It can be seen from Equation (4) that the RCS of the target is actually composed of two parts. First, the vector sum of scattered points in each range unit of the target is carried out to obtain the RCS of each range unit of the target, and then the scalar sum of all range units of the target is carried out to obtain the RCS of the whole target. 
In each measurement of the target RCS, its value is a certain but unknown constant, satisfying a $\chi^{2}$ distribution of $2 k$ degrees of freedom:

$$
p(\sigma)=\frac{k}{(k-1) ! \sigma_{0}}\left(\frac{k \sigma}{\sigma_{0}}\right)^{k-1} \exp \left(-\frac{k \sigma}{\sigma_{0}}\right), \sigma \geq 0
$$

Equation (5) gives the unified expression of the wideband radar $\chi^{2}$ distributed target model, whose distribution characteristics are determined by the double degree of freedom $K$, and the degree of freedom value is determined by the target type and the number of target range units. When $k=\infty$, the corresponding Swerling 0 type distribution target is represented as a non-undulation target, such as the calibration sphere. When $k=1$, it corresponds to Swerling I/II type distribution target, which is represented as the target with multiple scattering points with similar scattering intensity, and Swerling I is defined as slow fluctuation (correlated between pulses in a scan), Swerling II is defined as fast fluctuation (there is no correlation between pulses in a scan). When $k=2$, it corresponds to a Swerling III/IV-type distribution target, which is a target with multiple scattering points with similar scattering intensity and one strong scattering point. Swerling III is also defined as slow fluctuation; Swerling IV is defined as fast fluctuation. There are significant differences between a Swerling fast-fluctuation target and slow-fluctuation target in azimuth dimension, but not in distance dimension. Therefore, this paper takes the Swerling fast fluctuation target and the Swerling 0 type target as an example for analysis and research.

In addition, among the factors affecting the target distribution, the number of range units has a great influence on the distribution characteristics of Swerling II and Swerling IV. Of course, $m$ is closely related to the signal bandwidth, indicating that the RCS distribution of some targets is also related to the signal bandwidth. Although the five types of target models mentioned above cannot represent all the targets to be detected, they contain the most common radar targets, especially Swerling 0, Swerling II and Swerling IV distribution targets. This paper also builds target detection models based on this for analysis, and specific derivation and calculation will be given later.

\section{Wideband Radar Echo and Target Detection Model}

We used a wideband Linear Frequency Modulation (LFM) signal as the radar transmitting signal, and then preprocessed the received echo by orthogonal demodulation and matched filtering [17], used coherent/incoherent accumulation to complete the accumulation and finally the existence of the target was determined by threshold judgment. In order to clearly display the model established in this paper and the basic flow of specific operation steps, we established the following frame diagram (Figure 2):

\subsection{Wideband Signal Model}

The LFM signal is adopted as the transmitting signal and $N$ scattering points are assumed to exist on the target, then the echo received by each scattering point of the target can be expressed as:

$$
x(n)=\sqrt{a_{n}} \cos \left(-j 2 \pi f_{0}\left(t-t_{n}\right)+j \pi K\left(t-t_{n}\right)^{2}\right)+\omega(n), n=\mathbf{1}, \cdots, N
$$

where $a_{n}$ is the scattering point RCS, the echo amplitude is the square root value of the scattering point RCS, $f_{0}$ is the signal frequency, $K$ is the signal modulation frequency, $t$ is the fast time, $w(n)$ is the zero-mean complex Gaussian white noise with the variance of $\sigma^{2}$, $t_{n}$ is the target delay, which can be expressed as:

$$
t_{n}=\frac{2 r_{n}}{c}=\frac{2\left(r_{0}+r_{1}(n)\right)}{c}
$$


where, $r_{0}$ is the distance between target center and radar, $r_{1}$ is the distance between target scattering points and target center.

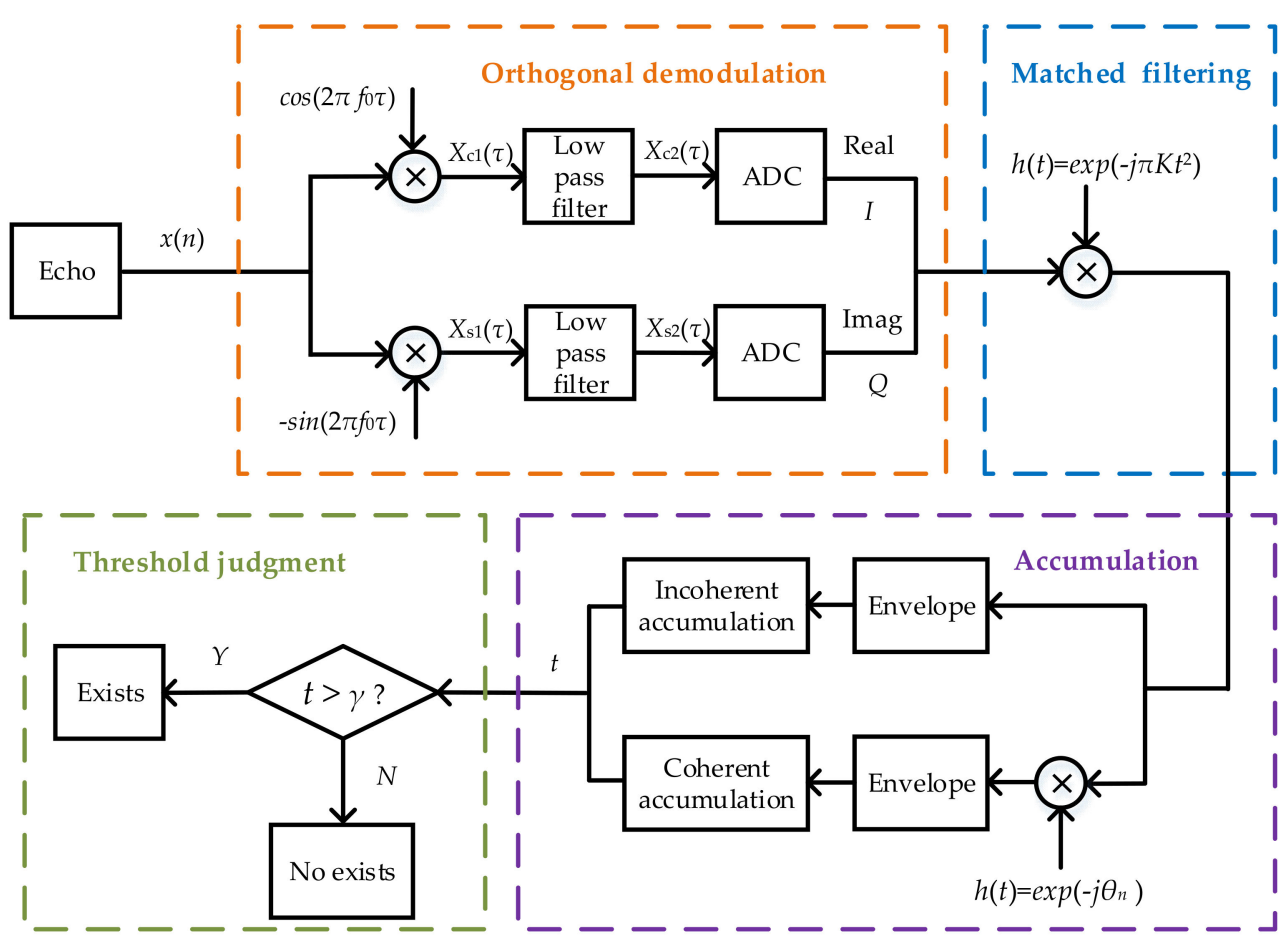

Figure 2. Wideband signal processing flow chart.

After orthogonal demodulation, the above equation can be rewritten as (See Appendix A for a detailed derivation):

$$
x(n)=\frac{\sqrt{a_{n}}}{2} \exp \left(-j 2 \pi f_{0} t_{n}+j \pi K\left(t-t_{n}\right)^{2}\right)+\widetilde{w}_{1}(n)
$$

where $w_{1}(n)$ is zero-mean white noise with variance of $\sigma^{2}$. Pulse compression is performed on Equation (8), and the matching filter function is:

$$
h(t)=\exp \left(-j \pi K t^{2}\right)
$$

After pulse compression, the following Equation can be obtained:

$x(n)=\frac{\sqrt{a_{n}} T}{2} \operatorname{sinc}\left(T K\left(t-t_{n}\right)\right) \exp \left(-j 2 \pi f_{0} t_{n}\right)+\widetilde{w}_{1}(n)=A(t) \exp \left(-j 2 \pi f_{0} t_{n}\right)+\widetilde{w}_{1}(n)$

The echo can be divided into coherent accumulation and non-coherent accumulation according to the different accumulation methods. Ideally, if the impulse response of the target is known in advance, we could match the receiving of the target echo, which is equivalent to coherent accumulation of each target-scattering echo, in which case the square envelope detector matching reception can be selected. The SNR after-echo accumulation is used as the test statistic, that is, the ratio of echo signal energy to noise energy. If the generalized likelihood ratio detector is selected [18], its detection statistic $t$ can be expressed as:

$$
t=\frac{2\left[\sum_{i=1}^{m}\left|x(i) \exp \left(-j \theta_{i}\right)\right|\right]^{2}}{m \sigma_{0}^{2}}
$$

where $\theta_{n}=2 \pi f_{0} t_{n}$ is the echo phase of the target scattering point. However, in practice, it is difficult to know the scattering information of the target in advance, so energy accumulation 
(incoherent accumulation) can only be carried out on the target during echo reception, and its detection statistic $t$ can be expressed as:

$$
t=\sum_{n=1}^{N} \frac{2|x(n)|^{2}}{\sigma^{2}}
$$

To determine the detection statistics, if the value is greater than the threshold, it is true; otherwise, it is false:

$$
t=\sum_{n=1}^{N} \frac{2|x(n)|^{2}}{\sigma^{2}} \underset{F}{<} \underset{F}{>} \gamma
$$

where, $\gamma$ is the threshold, its value depends on the false alarm probability, as defined below.

\subsection{Wideband Radar $\chi^{2}$ Distributed Target Detection Model}

SNR is the main factor determining target detection performance, so this section first analyzes the SNR of echo signal from radar equation. Suppose that in the transmission process of wideband radar, the input $\mathrm{SNR}$ is $\mathrm{SNR}_{0}$ :

$$
S N R_{0}=\frac{P_{t} G^{2} \lambda^{2}}{(4 \pi)^{3} k T_{0} B_{I F} F R^{4}} \cdot \sigma
$$

where $P_{t}$ is the radar transmitting signal power, which has been kept constant in the research and simulation experiment in this paper. $G$ is antenna gain, $\lambda$ is wavelength, $n$ is the number of irrelevant pulses, $k$ is the Boltzmann constant, $T_{0}$ is the equivalent noise temperature, $B_{I F}$ is the receiver bandwidth, $F$ is the receiver noise coefficient, and $R$ is the distance between the target and the radar.

After pulse compression, the SNR gain is:

$$
S N R_{1}=S N R_{\mathbf{0}} \cdot S N R_{D}=\frac{P_{t} G^{2} \lambda^{2} D \sigma}{(4 \pi)^{3} k T_{0} B_{I F} F R^{4}}=\mathrm{SNR}_{\mathrm{in}} \cdot \sigma
$$

According to the analysis, the target has $m$ range units, so it can generate $m$ different pulse signals. The target echo satisfies the non-central $\chi^{2}$ distribution with the degree of freedom of $2 m$ and the average signal-to-noise ratio as the non-central parameter. The probability density function can be expressed as:

$$
f(t)=\sum_{i=0}^{+\infty} \frac{t^{m+i-1}\left(S N R_{i n} \cdot \sigma\right)^{i} \exp \left\{\left(S N R_{i n} \cdot \sigma+t\right) / 2\right\}}{2^{m+i} \Gamma(m+i-1) i !}
$$

Taking the actual SNR of echo as the target detection statistic, the specific expression is shown in Equation (12), then the detection probability can be expressed as:

$$
P D=\int_{\gamma}^{+\infty} f(t) d t=\int_{\gamma}^{+\infty} \sum_{i=0}^{+\infty} \frac{t^{m+i-1}\left(S N R_{i n} \cdot \sigma\right)^{i} \exp \left\{\left(S N R_{i n} \cdot \sigma+t\right) / 2\right\}}{2^{m+i} \Gamma(m+i-1) i !} d t
$$

Integral operation of Equation (17) can be simplified as:

$$
P D=\exp \left\{-\frac{\gamma+S N R_{i n} \cdot \sigma}{2}\right\} \sum_{i=0}^{+\infty} \frac{1}{(i+m-1) !}\left(\frac{S N R_{i n} \cdot \sigma}{2}\right)^{i+m-1} \sum_{k=0}^{i} \frac{1}{k !}\left(\frac{\gamma}{2}\right)^{k}
$$




\subsection{Detection Model of Wide-Band Radar Swerling 0 Distributed Target}

The Swerling 0 type fluctuation distribution is also called undulating distribution, corresponding to $k=\infty$ in Equation (5). The target contains $N$ identical and independent scattering points, and the scattering point RCS is set as $a$. The expression of the target RCS is the same as Equation (2). The detection statistics of the target RCS are shown in Equation (12). Its non-central parameter is the average signal-to-noise ratio of echo, and the non-central parameter $\lambda$ can be expressed as:

$$
\lambda=2 \sigma \cdot S N R_{1}=\sum_{i=1}^{m} \sum_{j=1}^{n_{i}} a_{j} \cdot S N R_{1}=N a \cdot S N R_{1}
$$

The number of range units of the target is $m$, satisfies the chi-square distribution with $2 m$ degree of freedom and $\lambda$ as non-central parameter, and its probability density function can be expressed as:

$$
f_{T}(t)=e^{-t / 2} e^{-N a \cdot S N R_{1}} \sum_{i=0}^{+\infty} \frac{1}{i !}\left(N a \cdot S N R_{1}\right)^{i}\left(\frac{t^{i+m-1}}{2^{i+1}(i+m-1) !}\right), t>0
$$

Its detection probability can be expressed as:

$$
P D=\int_{\gamma}^{+\infty} f_{T}(t) d t=e^{-N a \cdot S N R_{1}} e^{-\frac{\gamma}{2}} \sum_{i=0}^{+\infty} \frac{\mathbf{1}}{i !}\left(N a \cdot S N R_{\mathbf{1}}\right)^{i} \sum_{k=\mathbf{0}}^{i+m-\mathbf{1}} \frac{\mathbf{1}}{k !}\left(\frac{\gamma}{\mathbf{2}}\right)^{k}
$$

where, $\gamma$ is the detection threshold, which is determined by false alarm probability $P_{f a}$, in this paper, the value of $P_{f a}$ is selected as $10 e^{-6}$, when the false alarm rate is determined, the value of the detection threshold can be obtained by Equation (22). In order to facilitate calculation, the detection threshold-false alarm probability curve can be drawn in advance.

$$
P_{f a}=e^{-\frac{\gamma}{2}} \sum_{i=1}^{m-1}\left(\frac{\gamma}{2}\right)^{i} \frac{1}{i !}
$$

\subsection{Detection Model of Wide-Band Radar Swerling II Distributed Target}

The Swerling I and Swerling II distribution targets are those that include multiple scattering points with independent and similar scattering intensities, also known as "exponential distributions" or "Rayleigh-power PDF". Where Swerling I is not correlated between scans and is called "scan-to-scan fluctuation" or "slow fluctuation"; Swerling II, which is correlated between scans and independent between pulses, is called "fast fluctuation." The signal studied in this paper is the incoherent accumulation gain problem of single-pulse signals, and the target is considered to be independent and irrelevant between the pulses of each range unit. Therefore, the Swerling II distributed target model is established for analysis in this paper.

For the Swerling II distribution target, the in-phase component and orthogonal component of each range element of the target echo are zero-mean Gaussian distribution, and the sum of the envelope satisfies Rayleigh distribution. $M$ is the number of range elements of the target, so the sum of the target echo follows a non-center chi-square distribution with a degree of freedom of $2 \mathrm{~m}$. The probability density function is the same as Equation (5), where $k=m$, and Equation (5) can be rewritten as:

$$
p(\sigma)=\frac{m}{(m-1) ! \sigma_{0}}\left(\frac{m \sigma}{\sigma_{0}}\right)^{m-1} \exp \left(-\frac{m \sigma}{\sigma_{0}}\right), \sigma \geq 0
$$


Suppose the scattering point intensity is a, and the average target RCS is expressed in Equation (4), then $\sigma_{0}$ can be expressed as:

$$
\sigma_{0}=\sum_{i=1}^{m} \sigma_{i}=a \cdot \sum_{i=1}^{m}\left|\sum_{j=1}^{n} \exp \left(\frac{4 \pi j R_{x}(j)}{\lambda}\right)\right|^{2}
$$

Equation (24) is substituted into Equation (23), and the detection statistic is shown in Equation (12), whose probability density function is similar to Equation (5) and can be expressed as:

$$
f(t)=\frac{m}{(m-1) ! \sigma_{0}}\left(\frac{m t}{\sigma_{0} \cdot S N R_{\text {in }}}\right)^{m-1} \exp \left(-\frac{m t}{\sigma_{0} \cdot S N R_{\text {in }}}\right), t \geq 0
$$

The detection threshold is set as $\gamma$, its definition is the same as Equation (22), and the detection probability can be obtained by substituting the detection threshold into Equation (25):

$$
P D=\int_{\gamma}^{+\infty} f(t) d t=\int_{\gamma}^{+\infty} \frac{m}{(m-1) ! \sigma_{0}}\left(\frac{m t}{\sigma_{0} \cdot S N R_{i n}}\right)^{m-1} \exp \left(-\frac{m t}{\sigma_{0} \cdot S N R_{i n}}\right) d t
$$

Integral operation of Equation (26) can be simplified as:

$$
P_{D}=\exp \left(-\frac{\gamma}{2\left(\sigma_{0} \cdot S N R_{i n}\right)}\right) \sum_{k=0}^{m-1} \frac{1}{k !}\left(\frac{\gamma}{2\left(\sigma_{0} \cdot S N R_{i n}\right)}\right)^{k}
$$

\subsection{Target Distribution Model of Wideband Radar Swerling IV Type}

Swerling III and Swerling IV distribution targets include a strong scattering point and multiple independent weak scattering points with similar scattering intensity, also known as the "Rician distribution". Swerling III is not correlated between scans and is called "scan-to-scan fluctuation" or "slow fluctuation". The Swerling IV is correlated between scans and independent between pulses, and is called "fast fluctuation." As mentioned above, the Swerling IV distribution target model is established in this section.

In fact, there are certain differences between the Swerling IV distribution target model and the Rician distribution model. The similar description of the Rician distribution is more specific and realistic, but the calculation is more complex. The probability density function can be expressed as follows:

$$
p(\sigma)=\frac{\sigma}{\sigma_{0}^{2}} \exp \left(-\frac{\sigma^{2}+v^{2}}{2 \sigma_{0}^{2}}\right) I_{0}\left(\frac{\sigma v}{\sigma_{0}^{2}}\right), \sigma \geq 0
$$

where $I_{0}(Z)$ is the modified zero-order Bessel function of the first class (see Appendix B for details), and the Rician distribution is usually described by parameter $K$, known as rice factor:

$$
K=\frac{v^{2}}{2 \sigma^{2}}
$$

The value of $K$ represents the weight of strong scattering points in the target. When $K=0$, the above Equation is equivalent to the Rayleigh distribution probability density function; when $K=+\infty$, the above Equation is equivalent to the undulating distribution. Considering the complexity of the Rician distribution, the $\chi^{2}$ distribution can be used for calculation.

Suppose that the weak scattering point RCS represents $a$, the strong scattering point RCS is $b$, the number of weak scattering points is $N$, and the target RCS is shown in 
Equation (4), then the scattering echo amplitude of the target within each range unit can be expressed as:

$$
\sigma_{i}=\left|\sum_{j=1}^{n_{i}} \sqrt{a} \exp \left(\frac{4 \pi j R_{x}(j)}{\lambda}\right)+\sqrt{b}\right|^{2}
$$

The target average RCS can be expressed as:

$$
\sigma_{0}=\sum_{i=1}^{m} \sigma_{i}=\sum_{i=1}^{m}\left|\sum_{j=1}^{n} \sqrt{a} \exp \left(\frac{4 \pi j R_{x}(j)}{\lambda}\right)+\sqrt{b}\right|^{2}
$$

The Swerling IV fluctuation distribution detector obeys the $\chi^{2}$ distribution with a degree of freedom of $4 m$, and its probability density function is similar to Equation (5), which can be expressed as:

$$
p(\sigma)=\frac{2 m}{(2 m-1) ! \sigma_{0}}\left(\frac{2 m \sigma}{\sigma_{0}}\right)^{2 m-1} \exp \left(-\frac{2 m \sigma}{\sigma_{0}}\right), \sigma \geq 0
$$

The detection threshold was set as $\gamma$ with Equation (22), and the detection probability was obtained by integrating Equation (32):

$$
P D=\int_{\gamma}^{+\infty} f(t) d t=\int_{\gamma}^{+\infty} \frac{2 m}{(2 m-1) ! \sigma_{0}}\left(\frac{2 m t}{\sigma_{0} \cdot S N R_{\text {in }}}\right)^{m-1} \exp \left(-\frac{2 m t}{\sigma_{0} \cdot S N R_{\text {in }}}\right) d t
$$

After calculation and simplification, Equation (33) can be rewritten as:

$$
P_{D}=\exp \left(-\frac{\gamma}{2\left(\sigma_{0} \cdot S N R_{i n}\right)}\right)^{2 m-1} \frac{1}{k=0}\left(\frac{\gamma}{2\left(\sigma_{0} \cdot S N R_{i n}\right)}\right)^{k}
$$

\section{Diversity Gain of Wideband Radar}

The traditional radar equation is only applicable to a single-pulse signal, and the influence of accumulation loss and fluctuation loss must be taken into account when incoherent accumulation of a multi-pulse signal is carried out. In practice, incoherent accumulation not only exists between pulses, but also between different distance elements within pulses. The corresponding incoherent accumulation loss and fluctuation loss are also numerically the same.

\subsection{Diversity Gain Definition}

As can be seen from the above analysis, with the increase in radar signal bandwidth, the range resolution of radar also increases, and the discernable range unit increases. As shown in Figure 3, on the one hand, the number of target echo incoherent accumulation increases, and the incoherent accumulation loss increases, which leads to the reduction in the SNR of output accumulation.

The incoherent accumulation efficiency was defined as $E$, and the loss caused by signal bandwidth on target echo accumulation was quantitatively analyzed by using this variable, the fluctuation loss is $L$, which is used to analyze the influence of signal bandwidth on the fluctuation of target RCS. In addition, the RCS of each range unit of the target is also decreasing, and the fluctuation of the overall RCS of the target will also decrease. In general, incoherent accumulation efficiency $E$, undulating loss $L$ and target RCS are used to uniformly describe the impact of bandwidth on target detection performance. When the relation between bandwidth $B$ and initial bandwidth $B_{0}$ (the corresponding bandwidth when the target has only one resolution unit) is $m$ times, it can be expressed as:

$$
P D(B)=P D\left(m B_{0}\right)=f(E(m), 1 / L(m), R C S(m))=f\left(n_{1}(m), p(m), n_{2}(m)\right)
$$




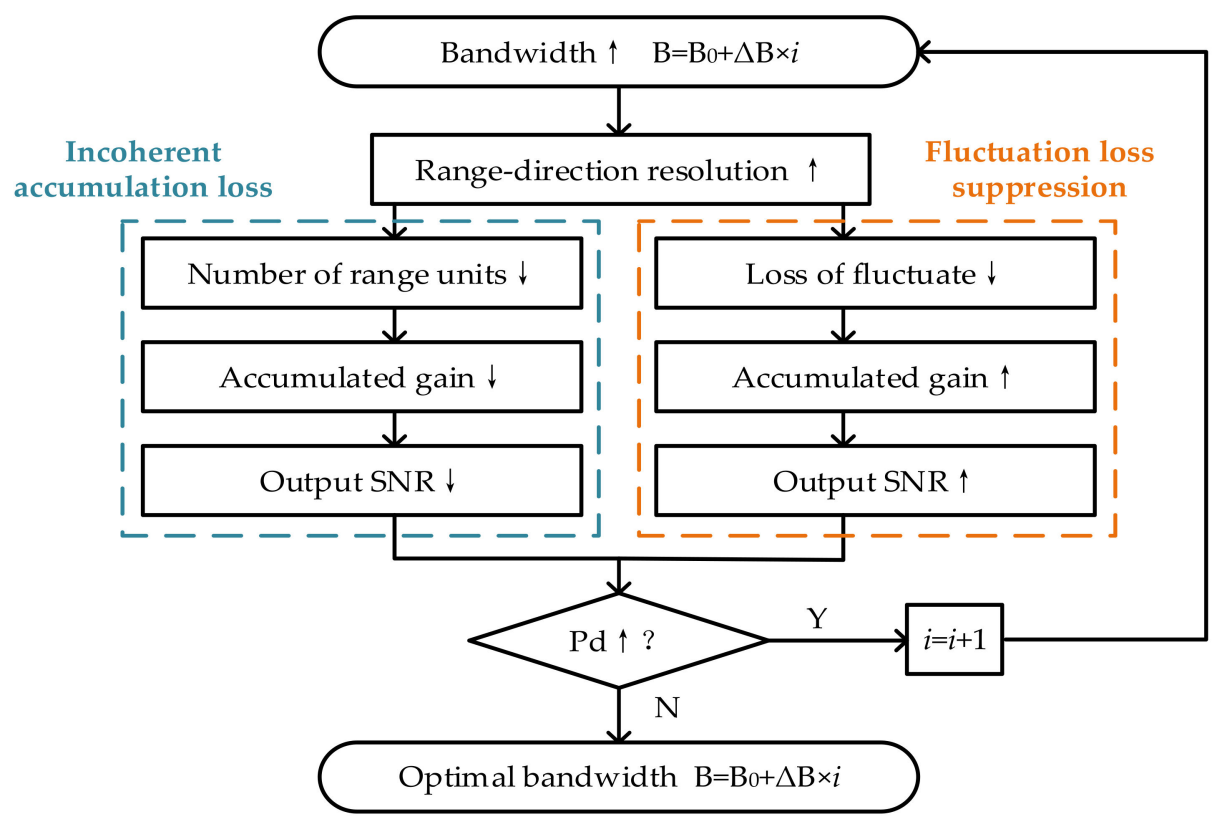

Figure 3. Diversity gain flow chart of wideband radar.

As has been discussed above, we give the definition of generalized frequency diversity gain: there is a dynamic relationship between the detection performance of the target and the number of distinguishable range units of the target. When the target has only one range unit, the corresponding detection performance is the reference benchmark. The ratio of the corresponding detection performance to the reference when the target is resolved into multiple range units is defined as diversity gain, which can be expressed as:

$$
E\left(m B_{0}\right)=\frac{E(m) R C S(m) L(1)}{E(1) R C S(1) L(m)}
$$

When the detection performance is optimal or the SNR gain is maximum, the corresponding diversity gain is optimal diversity gain.

\subsection{Diversity Gain of Incoherent Accumulation for Wideband Radar}

Conventional wisdom says that due to the non-linearity of the geophone, incoherent accumulation has accumulation loss and the incoherent accumulation gain of $n_{e}$ pulse is smaller than that of $n_{e}$. Traditional experience believes that when $n_{e}$ is large, the gain effect is approximately $n_{e}{ }^{1 / 2}$.

Extended by Albersheim's empirical Equation, the gain of incoherent accumulation can be approximately expressed as [19]:

$$
(S / N)_{n_{e}}=-5 \ln n_{e}+\left(6.2+\frac{4.54}{\sqrt{n_{e}-0.44}}\right) \times \lg (A+0.12 A B+1.7 B)
$$

where, $A=\ln \left(0.62 / \mathrm{P}_{\mathrm{fa}}\right), B=\ln \left[\mathrm{P}_{\mathrm{d}} /\left(1-\mathrm{P}_{\mathrm{d}}\right)\right], n_{e}$ is the number of effective independent samples. The error of this equation is less than $0.2 \mathrm{~dB}$ in the range of $\mathrm{n}=1 \sim 8096, \mathrm{P}_{\mathrm{d}}=0.1 \sim 0.9$, $\mathrm{P}_{\mathrm{fa}}=10^{-3} \sim 10^{-7} . E_{i}$ is defined as the accumulation efficiency of incoherent accumulation, which can be expressed as:

$$
E_{i}\left(n_{e}\right)=\frac{(S / N)_{1}}{n_{e}(S / N)_{n_{e}}}
$$


Substitute Equation (38) into Equation (15) to obtain the following equation

$$
S N R=S N R_{1}+S N R_{E}=10 \log _{10}\left(\frac{P_{t} G^{2} \lambda^{2} T \sigma n_{e} E_{i}\left(n_{e}\right)}{(4 \pi)^{3} k T_{0} F\left(L_{f}\right)^{1 / n e} R^{4}}\right)
$$

where, $L(f)$ is the fluctuation loss, which can be expressed as:

$$
L_{f}\left(n_{e}\right)=\left(L_{f}\right)^{1 / n_{e}}
$$

It can be seen from Equation (40) that the fluctuation loss decreases with the increase in $n_{e}$; that is, incoherent accumulation can effectively suppress the fluctuation degree of echo.

\subsection{Incoherent Accumulation Gain of a Wideband Radar Undulating Target}

According to the radar equation, multiple variables can affect the final signal output SNR: signal transmitting power, signal frequency (wavelength), target RCS, antenna gain, receiver bandwidth, distance between target and radar, etc. In order to analyze the direct relationship between bandwidth and target detection probability, the signal transmitting power, signal frequency and antenna gain are kept constant, and the receiver bandwidth is equal to the transmitting bandwidth. Therefore, the only factors that determine signal SNR are target RCS, pulse accumulation mode, number of independent pulses and distance. Therefore, Equation (39) can be rewritten as:

$$
S N R=10 \log _{10}\left(\frac{P_{t} G^{2} \lambda^{2} T}{(4 \pi)^{3} k T_{0} F}\right)+10 \log _{10}(\sigma)+10 \log _{10}\left(\frac{n_{e} E_{i}\left(n_{e}\right)}{\left(L_{f}\right)^{1 / n_{e}}}\right)-10 \log _{10}\left(R^{4}\right)
$$

The target RCS is related to the signal bandwidth, and the larger the bandwidth, the higher the radar range resolution. The smaller the size of each range unit, the smaller the corresponding target RCS fluctuations, satisfying the Swerling distribution. The number of independent pulses is also related to the signal bandwidth. The larger the bandwidth, the more independent samples of the target echo from the distance upwards, the lower the incoherent accumulation gain efficiency and the lower the fluctuation loss. In addition, distance is also an independent variable that affects the size of SNR and it is independent of the signal bandwidth.

The following equation is the part of SNR related to bandwidth in Equation (41):

$$
S N R_{B}=10 \log _{10}(\sigma)+10 \log _{10}\left(\frac{n_{e} E_{i}\left(n_{e}\right)}{\left(L_{f}\right)^{1 / n_{e}}}\right)
$$

Rcs is defined in Equations (24) and (31), corresponding to Swerling II and Swerling IV targets, respectively. With the increase in bandwidth, when the result of the above equation is positive (logarithmic value $>1$ ), it indicates that SNR increases with the increase in bandwidth. When the result of the above equation is zero, it indicates that the critical bandwidth has been reached.

As shown in Figure 4, the abscissa is the number of range units of the target, and the ordinate is the diversity gain efficiency. The figure shows the total incoherent accumulation efficiency of wideband radar with different bandwidth under different detection probability requirements. It can be seen that the initial wideband diversity gain efficiency of the target is one, which means that there is no accumulation loss and fluctuation loss suppression when the target has only one range unit. For Swerling II-type targets, when the detection probability requirement is above $80 \%$, the targets should be divided into $4-5$ range units. When the detection probability requirement reaches more than $95 \%$, the target needs to be 
resolved into 6-8 range units; For Swerling IV-type targets, when the detection probability requirement is above $80 \%$, the targets need to be resolved into multiple range units.

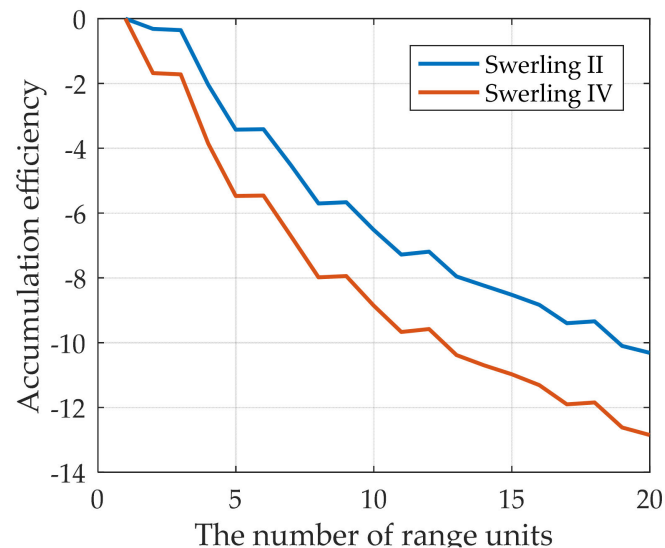

(a)

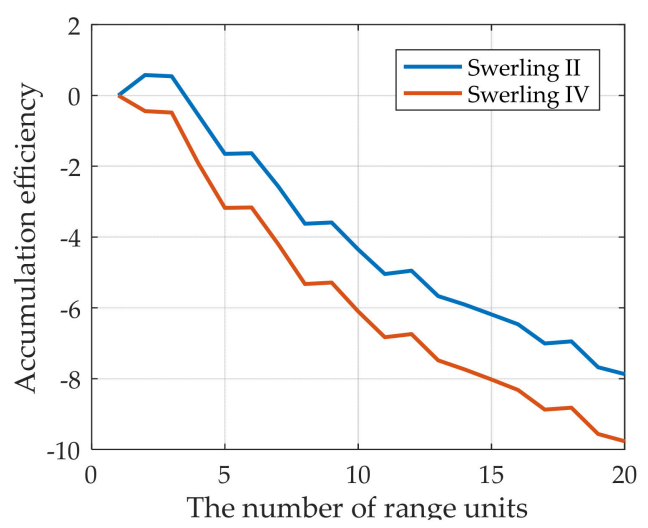

(c)

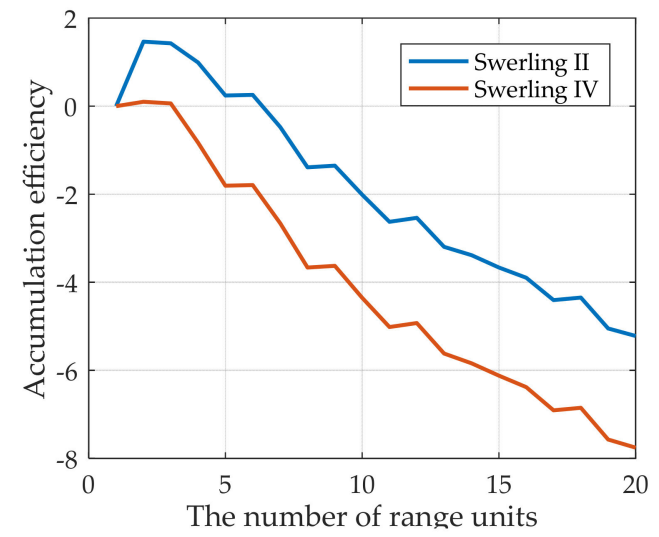

(e)

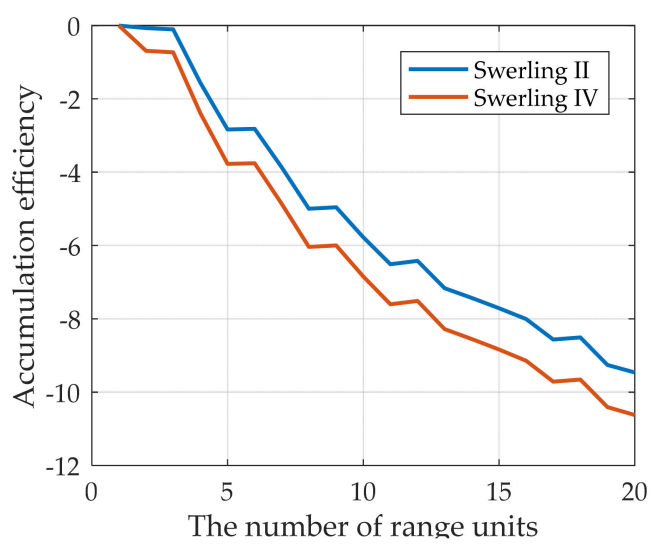

(b)

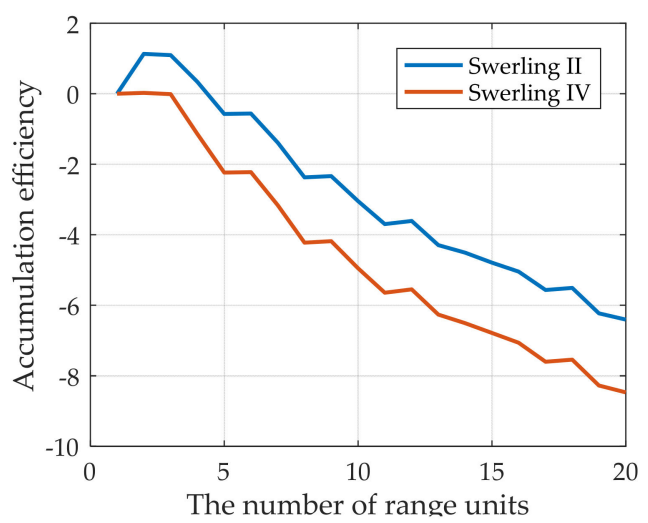

(d)

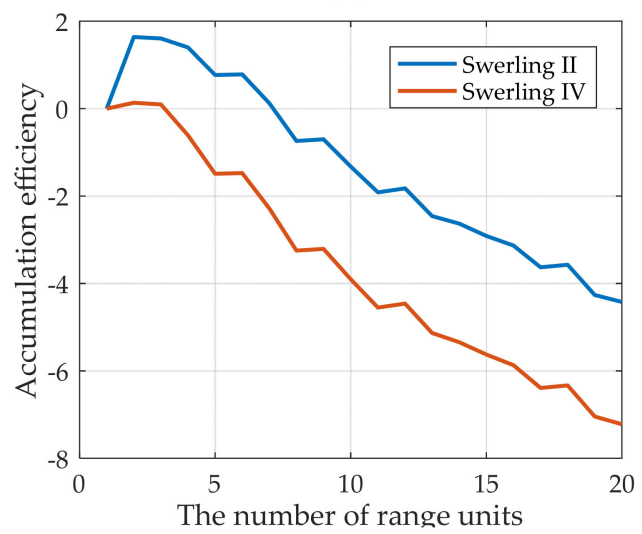

(f)

Figure 4. Diversity gain diagram of wideband radar (a) $\mathrm{P}_{\mathrm{d}}=0.5 ;(\mathbf{b}) \mathrm{P}_{\mathrm{d}}=0.6$; (c) $\mathrm{P}_{\mathrm{d}}=0.7 ;(\mathbf{d}) \mathrm{P}_{\mathrm{d}}=0.8$; (e) $\mathrm{P}_{\mathrm{d}}=0.9$; (f) $\mathrm{P}_{\mathrm{d}}=0.95$.

There will be differences between the actual target and the model established in this paper, but as long as the target has a certain fluctuation, this phenomenon will occur, but the degree will be different. Therefore, the theoretical thinking in this paper can satisfy all types of targets. As long as the distribution function of the fluctuation characteristic of the target can be given, the optimal number of range units of the target can be calculated. 


\section{Simulation Experiment Verification and Analysis}

\subsection{Simulation Experiment Parameter Setting}

We explore the relationship between signal bandwidth and target detection probability through the control variable method, but it is obvious that the relationship between the two variables is not linearly invariant. According to the above analysis, the increase in signal bandwidth decreases the SNR of the echo signal and the fluctuation degree of the echo signal, which presents a mutual restriction effect on target detection. When the two reach a balance, the corresponding signal bandwidth is the optimal detection bandwidth.

Specific simulation parameters are set as follows (Table 1):

Table 1. Simulation experiment parameters.

\begin{tabular}{ccc}
\hline Parameters & Symbol & Value \\
\hline Radar peak power & $P_{t}$ & $100 \mathrm{KW}$ \\
Antenna gain & $G$ & $24 \mathrm{~dB}$ \\
The signal bandwidth & $B$ & $200 \mathrm{MHz}$ \\
Signal carrier frequency & $f_{c}$ & $1.5 \mathrm{GHz}$ \\
Receiver bandwidth & $B_{I F}$ & $200 \mathrm{MHz}$ \\
Pulse duration & $T$ & $50 \mathrm{us}$ \\
Time-bandwidth product & $D$ & 400 \\
Noise factor & $F_{n}$ & $3 \mathrm{~dB}$ \\
System loss & $L_{n}$ & $15 \mathrm{~dB}$ \\
Initial target distance & $R_{0}$ & $50 \mathrm{Km}$ \\
The target size & $L$ & $40 \mathrm{~m}$ \\
Target scattering point & $N$ & 20,000 \\
False alarm rate & $P_{f a}$ & $10^{-6}$ \\
\hline
\end{tabular}

A group of experiments were designed, each of which adopted different signal bandwidth. Firstly, random and unknown targets are generated according to the fluctuation characteristics of the target. There are 20,000 scattering points in the target, which are evenly distributed in each range element. The target RCS of each range element is the sum of the scattering points in the range element. The echo of all range units of the target is incoherent accumulation, the detection threshold is obtained by the calculation of false alarm probability and the detection is completed by the threshold judgment.

In each group of experiments, the independent variable was the original SNR of the signal, and the dependent variable is target detection probability. According to radar equation theory, the input SNR of echo is closely related to transmitting power and antenna gain, etc. Therefore, in the experiment of this paper, such parameters are set as fixed reasonable values to ensure that the input SNR is only related to bandwidth at the same distance.

Different from [20-22], the wideband target model established in this paper includes a large number of scattering points. In traditional simulation methods, it is customary to set the target as a collection of a number of strong scattering points, or only one scattering point exists in each range unit of the target. In fact, the target is composed of numerous scattering points. Therefore, this paper assumes that the target has 20,000 scattering points. Even if the radar resolution is high, there are still a certain number of scattering points in each range unit, which is more realistic than the traditional method.

\subsection{Simulation Results and Analysis of Swerling 0 Target}

The Monte Carlo simulation is performed on the Swerling 0 distribution target, and the number of simulation experiments is 1000 . For the convenience of representation and comparison, the detection performance curves of targets under different bandwidths are put on a graph. The latter two goals are expressed in the same way. The simulation results are shown in Figure 5: 


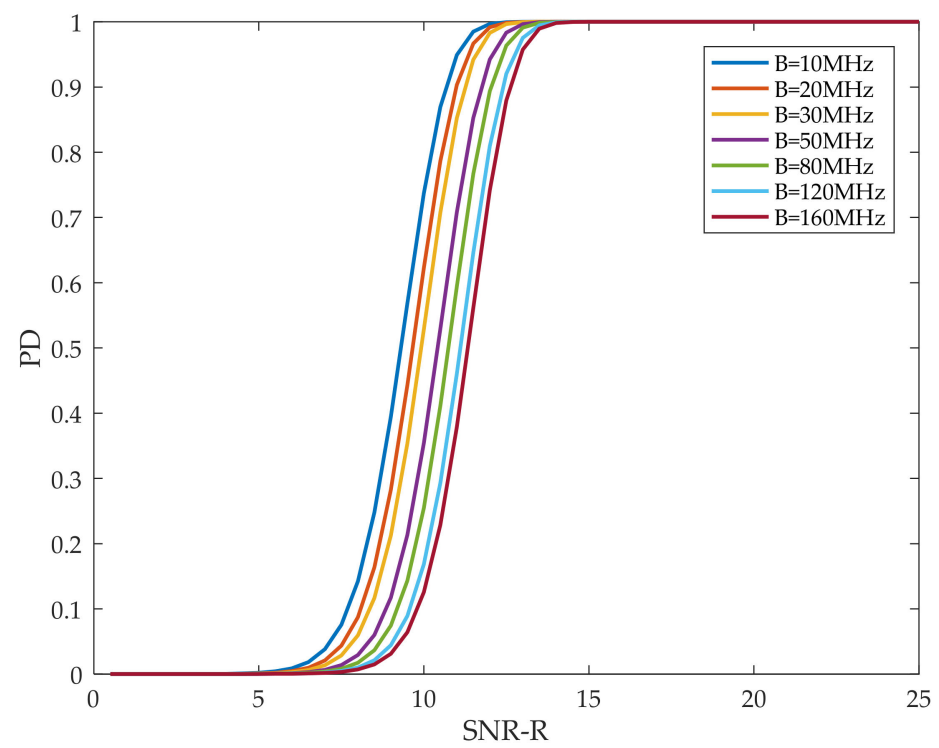

Figure 5. Swerling 0 distributed target-detection performance curve.

Figure 5 shows the radar detection performance of Swerling 0 distributed targets under different bandwidths, where the horizontal coordinate is the input SNR under the range dominance, that is, the SNR after pulse compression. At the same distance, all radar parameters except signal bandwidth are at the same level. In this case, signals with different bandwidth will affect the final detection result from receiving the SNR gain of subsequent processing. It can be seen that in the whole detection probability interval, the detection performance of the target gradually decreases with the increase in bandwidth.

However, the scattering points contained in the Swerling 0 distribution target are unfluctuating, and the fluctuation loss suppression caused by incoherent accumulation can be ignored, but the gain loss of incoherent accumulation increases with the increase in bandwidth. Therefore, the phenomenon of a high detection probability interval wideband being superior to narrow-band does not appear in Figure 5.

\subsection{Simulation Results and Analysis of Swerling II Target}

Monte Carlo simulation is performed on the Swerling II distribution target. The simulation results are as follows (Figure 6):

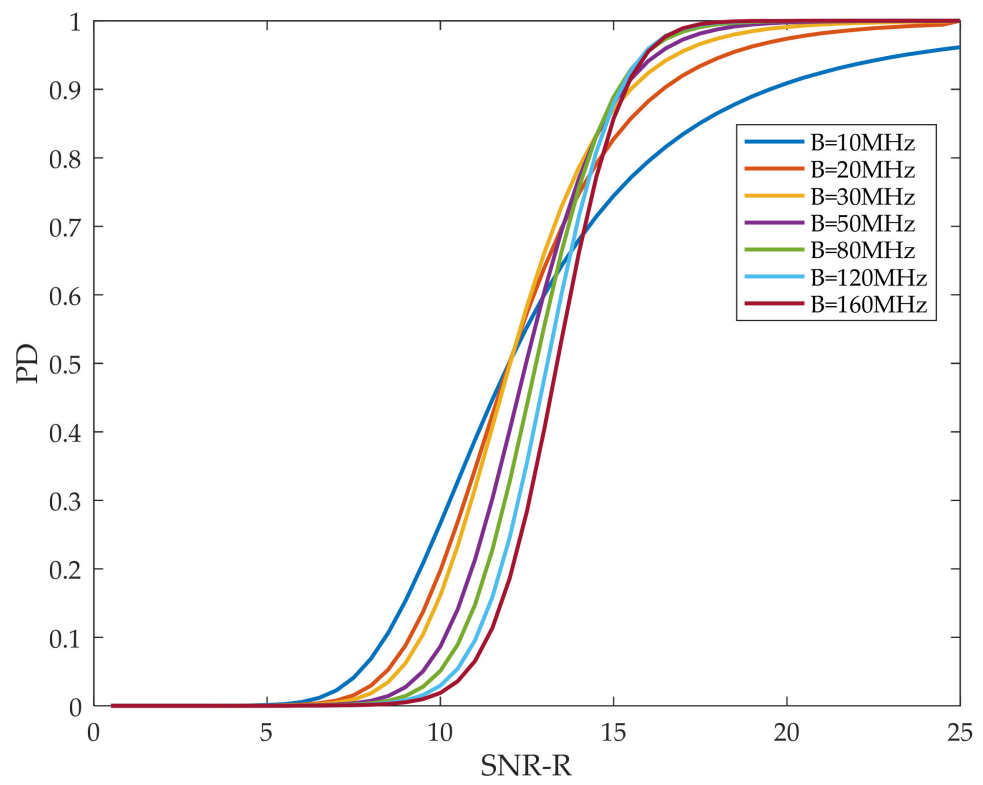

Figure 6. Swerling II distributed target detection performance curve. 
Figure 6 shows the radar detection performance of targets under different bandwidths, and the coordinate information remains unchanged as shown in Figure 5. It can be seen that when the signal bandwidth increases, the detection performance of radar becomes worse in the low-detection probability interval, whereas in the high-detection probability interval, the detection performance initially becomes better and then becomes worse, indicating that there is an optimal value of signal bandwidth in this detection probability interval.

According to the experiment of the Swerling 0 target, the detection performance of wideband radar on an undulating target is worse than that of narrowband radar, because the gain loss of incoherent accumulation increases with the increase in bandwidth. As for the Swerling II target, the wideband radar has a better detection probability in the high detection probability region, so it can be determined that the fluctuation loss gain performance of wideband radar increases with the increase in detection probability, which is consistent with the theoretical result and verifies the theoretical derivation in this paper.

Each curve in Figure 7 represents the detection performance of radar at different distances and bandwidths. When the distance is long, the corresponding input SNR is small, and the radar detection probability decreases with the increase in bandwidth. When the distance is small, the radar detection probability increases first and then decreases with the increase in bandwidth. The two phenomena are gradually changed with the increase in distance, and the detection probability is not completely synchronized with the increase in bandwidth with the decrease in distance.

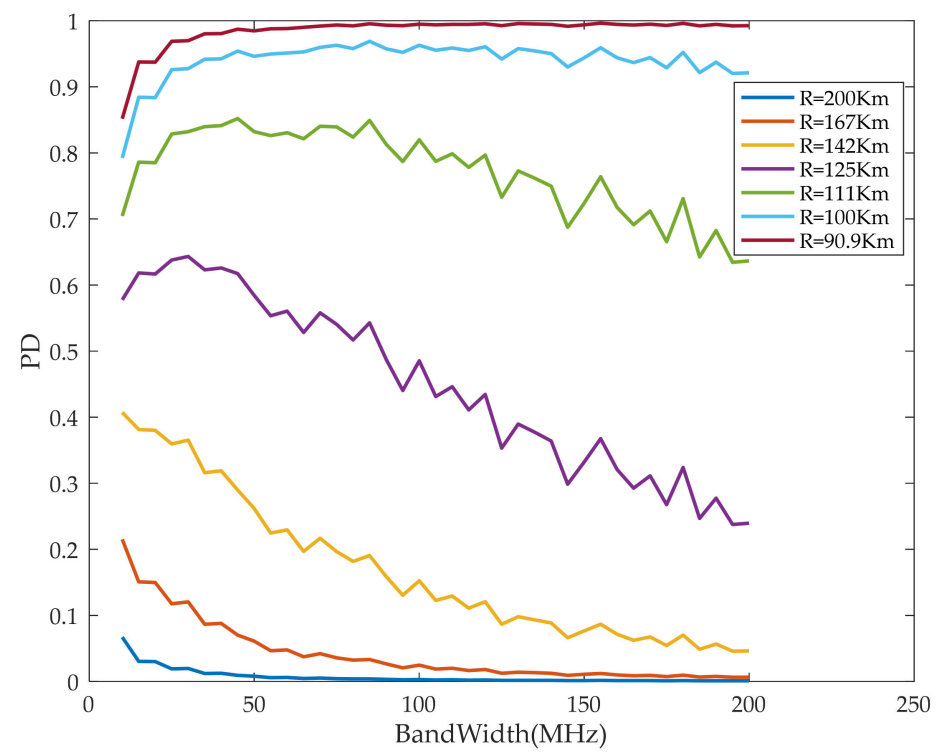

Figure 7. Target detection probability—bandwidth diagram at different distances.

Figure 8 represents the number of target range units under different detection probability requirements, that is, the bandwidth corresponding to the minimum SNR (the maximum detection distance) required for target detection under specific detection probability requirements. When the detection probability requirement is $70 \%$ or above, the target needs to be resolved into multiple range units. The simulation experiment results in the figure are consistent with the theoretical calculation results, which verifies the theoretical derivation process of incoherent accumulation gain of the wideband radar equation in this paper. It also proves the correctness of the derivation of detection performance curve of Swerling II-distributed targets under wideband radar. 


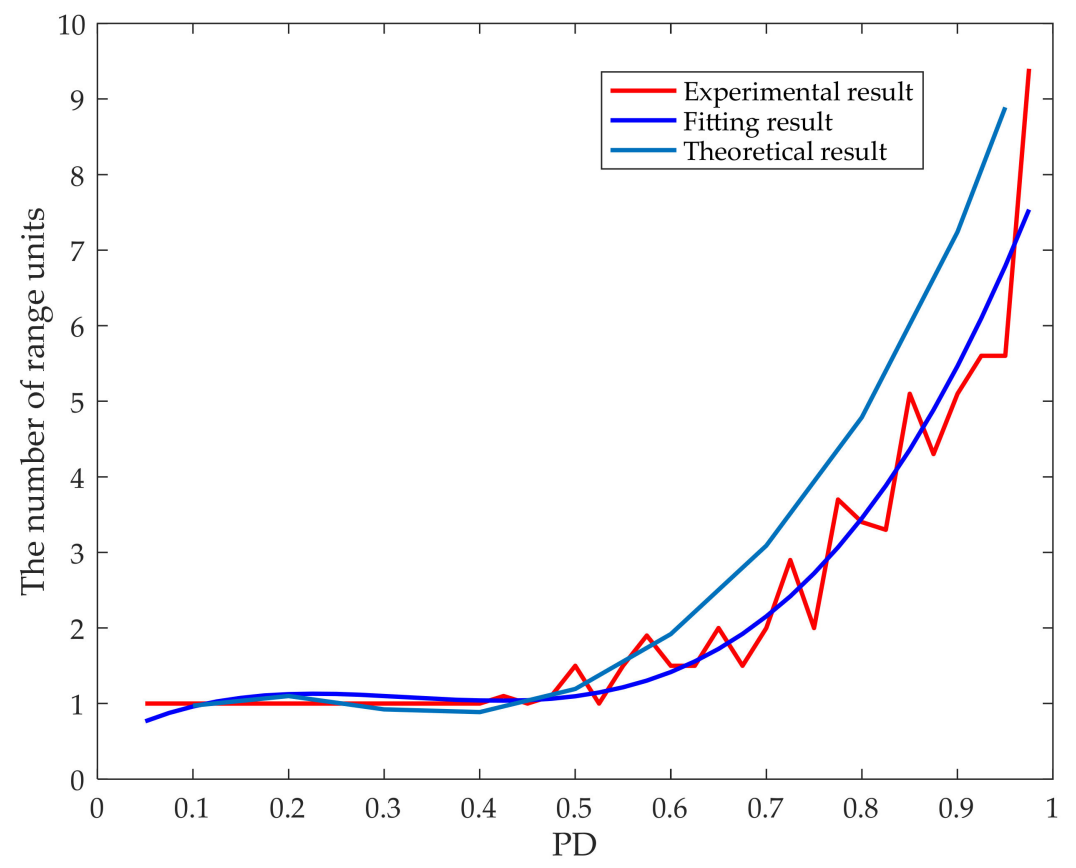

Figure 8. Swerling II distributed target detection performance curve.

Table 2 shows the number of range units under different detection probability requirements of Swerling II distribution targets, which are theoretical values, fitting values and simulation values, respectively. The data in the table are rounded off.

Table 2. Number of optimal range units in the Swerling II distribution.

\begin{tabular}{cccccc}
\hline Target & $\mathbf{P}_{\mathbf{d}}=\mathbf{0 . 6}$ & $\mathbf{P}_{\mathbf{d}}=\mathbf{0 . 7}$ & $\mathbf{P}_{\mathbf{d}}=\mathbf{0 . 8}$ & $\mathbf{P}_{\mathbf{d}}=\mathbf{0 . 9}$ & $\mathbf{P}_{\mathbf{d}}=\mathbf{0 . 9 5}$ \\
\hline Experimental & 2 & 2 & 3 & 5 & 8 \\
Fitted value & 1 & 2 & 3 & 5 & 7 \\
Theoretical value & 2 & 3 & 4 & 6 & 9 \\
\hline
\end{tabular}

\subsection{Simulation Results and Analysis of Swerling IV Target}

The design experiment was the same as above. RCS of the strong scattering point in the Swerling IV-type target was set as a, and the sum of the energy of the weak scattering point was set $\mathrm{as} b$ and $\mathrm{a}=\mathrm{b}$. See Table 1 for other parameters. The following results were obtained (Figure 9):

Figure 9 is similar to Figure 6, but the correlation between detection probability and bandwidth is significantly weaker than Figure 6, indicating that the incoherent accumulation of Swerling IV-type targets has a poor inhibition effect on fluctuation loss. In the high-detection probability interval, the detection probability quickly reaches the maximum as the bandwidth increases, and the optimal bandwidth is less than the Swerling II-type target under the same conditions. However, compared with Swerling 0 target, it can be found that the fluctuation loss of Swerling IV target is suppressed by broadband signal in the high-detection probability interval. Similarly, the suppression effect of wideband radar on the undulating target depends on the undulating degree of the target and improves with the increase in the undulating degree of the target. Therefore, the optimal bandwidth selection of the fluctuation target is closely related to its fluctuation degree. 


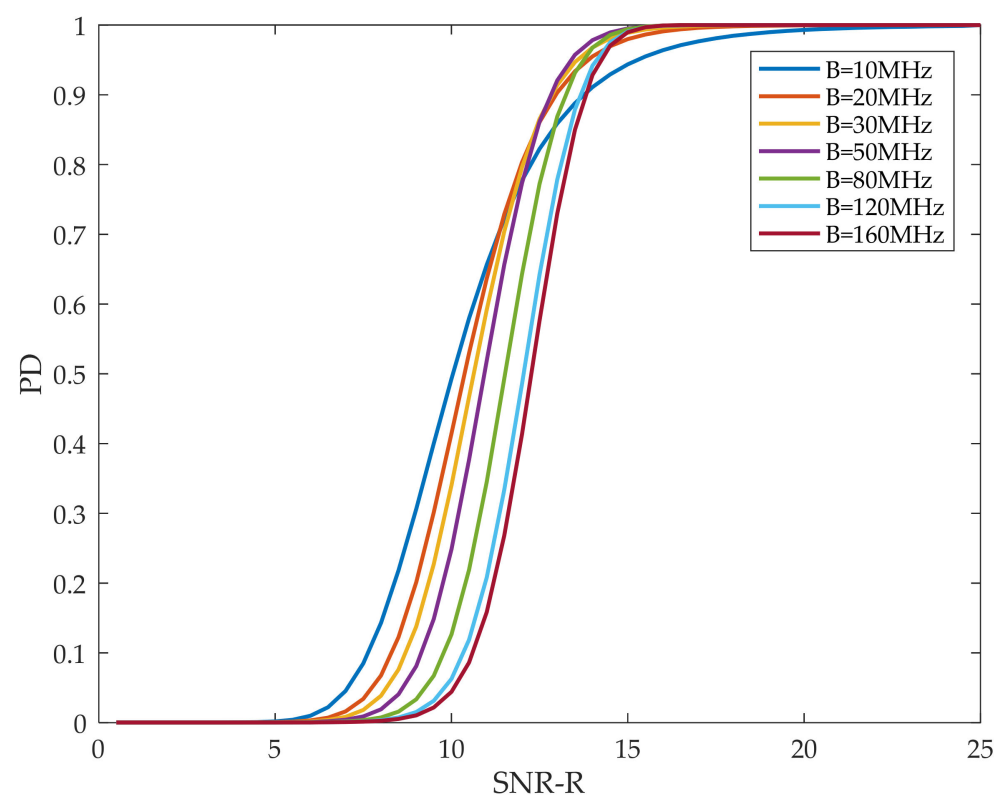

Figure 9. Swerling II-distributed target detection performance curve.

Figure 10 shows the detection capability of signals with different bandwidths for Swerling IV-type targets. The basic performance is similar to Figure 7 . The detection probability also increases first and then decreases in the interval of high SNR (near range interval).

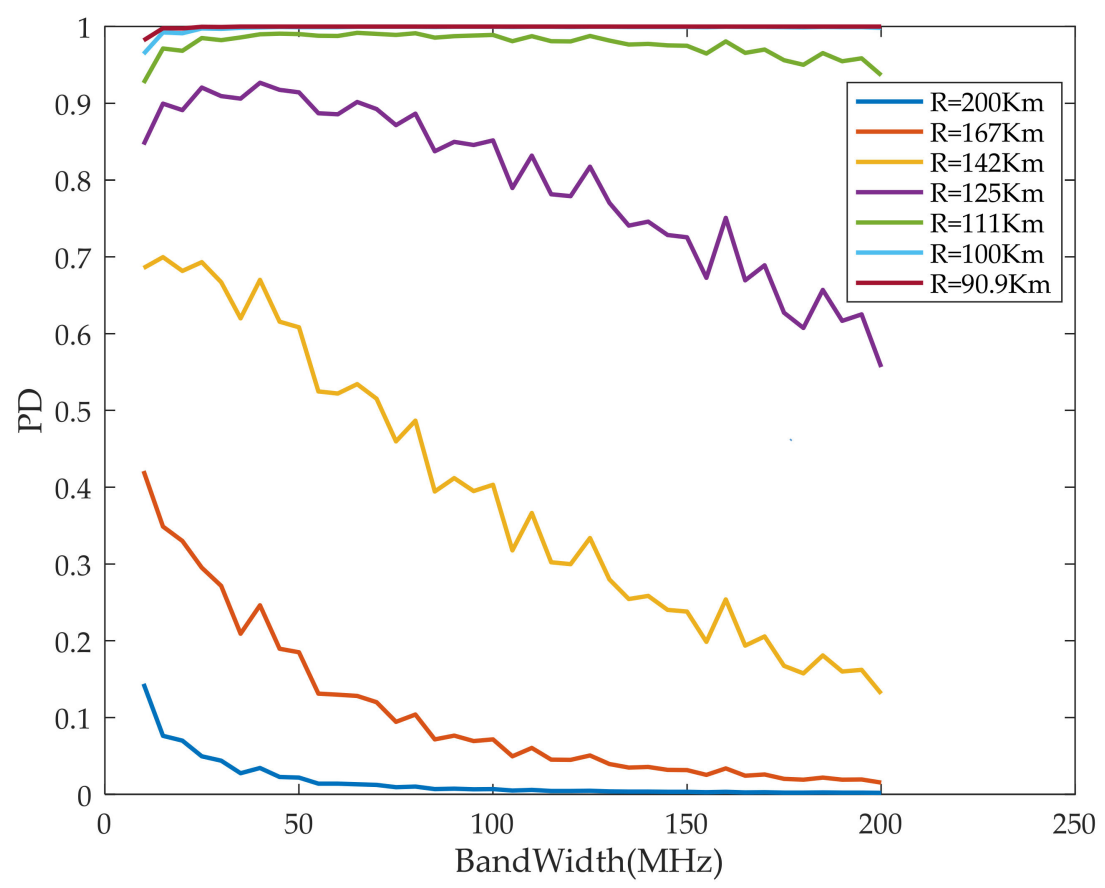

Figure 10. Swerling II distributed target detection performance curve.

Figure 11 shows the number of range units of Swerling IV-type targets under different detection probability requirements. When the detection probability requirement is $80 \%$ or above, the target needs to be resolved into 2-3 range units. The simulation experiment results in the figure are consistent with the theoretical calculation results, and the theoretical derivation is also verified. 


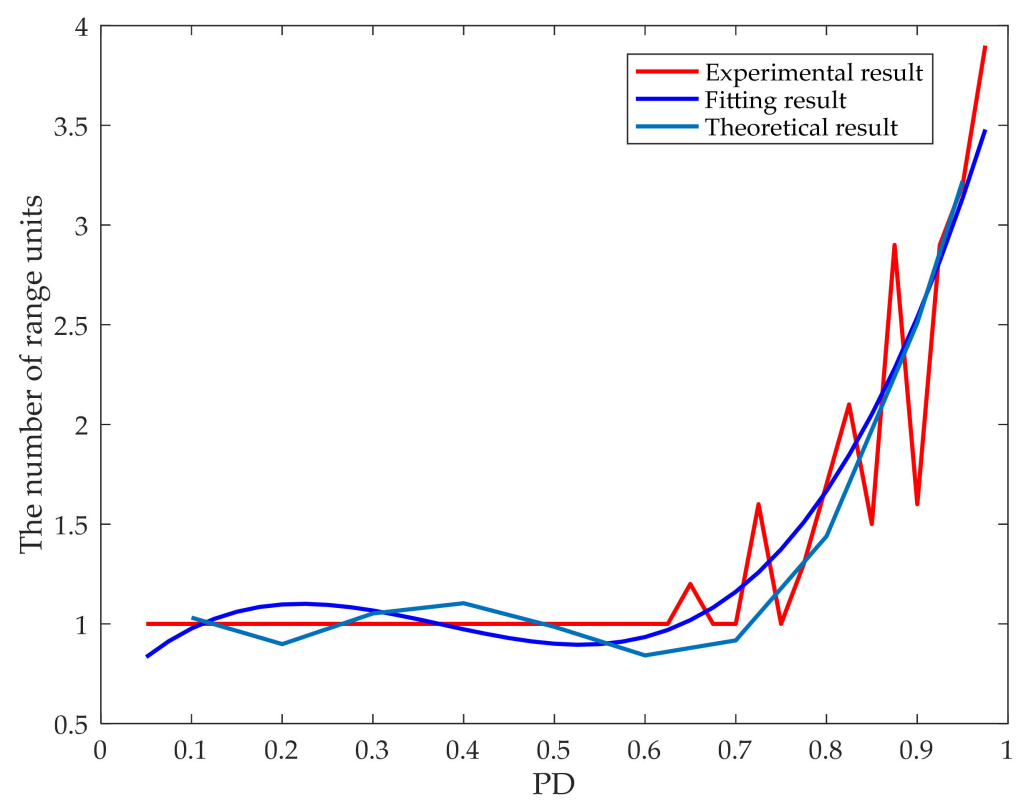

Figure 11. Swerling II distributed target detection performance curve.

Table 3 shows the number of range units under different detection probability requirements of Swerling IV distribution targets, which are theoretical values, fitting values and simulation values, respectively. Because values are generally small, none of the values in the table are rounded.

Table 3. Number of optimal range units in the Swerling IV distribution.

\begin{tabular}{cccccc}
\hline Target & $\mathbf{P}_{\mathbf{d}}=\mathbf{0 . 7}$ & $\mathbf{P}_{\mathbf{d}}=\mathbf{0 . 8}$ & $\mathbf{P}_{\mathbf{d}}=\mathbf{0 . 8 5}$ & $\mathbf{P}_{\mathbf{d}}=\mathbf{0 . 9}$ & $\mathbf{P}_{\mathbf{d}}=\mathbf{0 . 9 5}$ \\
\hline Experimental & 1 & 1 & 2 & 2 & 3 \\
Fitted value & 1 & 1 & 2 & 2 & 3 \\
Theoretical value & 1 & 1 & 2 & 2 & 3 \\
\hline
\end{tabular}

\subsection{Comparison and Analysis of Experimental Results}

It is given in reference [16] that for a $15 \mathrm{~m}$ target, when the detection probability is 0.8 , it should be distinguished into 5-10 range units. As shown in Table 2, when the target detection probability requirement is 0.8 , the Swerling II target should be resolved into 3-4 range units, and the Swerling IV target should be resolved into 1-2 range units. This is different from the literature [16]. For the convenience of analysis, the number of target range units is defined as the accumulation scale.

(1) The interval of the optimal accumulation scale given in this paper is smaller and more accurate. Different targets have different undulating characteristics, and the undulating loss gain brought by incoherent accumulation is also different, so the effect of diversity gain is also different, and the optimal accumulation scale will change accordingly. On the whole, the number of optimal target scales increases with the increase in target undulation.

(2) The optimal scale (2) in this paper, the results show that the target number and is not related to the target size, but related to the fluctuation characteristics of the target, and the number of range units of the target depends on the size of the target and signal bandwidth. Targets with different sizes correspond to different optimal signal bandwidths. However, for targets with the same distribution, different target sizes have nothing to do with the optimal accumulation scale.

Theoretical derivation and a series of simulation experiments show that, in the highdetection probability interval, wideband radar does have better detection performance 
than narrowband radar for undulating targets, but it cannot be considered that wideband radar has better detection performance than narrowband radar.

First of all, when the detection probability requirement is known, the number of optimal range units of undulating targets is fixed, so the optimal bandwidth is directly related to the target size. For large-size targets, radar with $20 \mathrm{MHz}$ bandwidth may be able to distinguish multiple range units. For small-size targets, a bandwidth of $100 \mathrm{MHz}$ may not be able to distinguish the target into multiple range units.

Secondly, the problem studied in this paper is the integration of target echo in range direction during a single scan, and the slow time dimension (azimuth integration) has not been involved in this paper. These two kinds of accumulation are ways to improve the detection performance of target echo. When the two are combined, more complex problems will appear, and more factors need to be considered to determine the optimal bandwidth. In reference $[23,24]$, several classical radar target azimuth echo accumulation methods are given. Combining the theory studied in this paper with such methods, the diversity gain of azimuth can be further studied.

Thirdly, the diversity gain effect of targets with different fluctuation types is different. The greater the fluctuation degree of targets, the more obvious the diversity gain effect is. Therefore, the advantages of wideband radar are also greater, otherwise the reverse is true. As Swerling 0 targets introduced in this paper do not have fluctuations, so the detection performance of wideband radar is worse than that of narrowband radar.

Finally, the theoretical analysis and simulation experiments in this paper are ideal, especially the setting of the target model. In practice, the target and its scattering point distribution are more complicated, so the calculation of the number of optimal range units is also more complicated. With the change of its fluctuation, the number of optimal range units is also changing.

\section{Conclusions}

This paper mainly studies the diversity gain of wideband radar. Firstly, according to the actual working principle of radar, we established the signal transmitting and receiving model and deduced the SNR gain problem in the working process of radar from the radar equation. Secondly, we accumulated the signal after pulse compression, and analyzed and studied the coherent accumulation and incoherent accumulation. A wide band radar $\chi^{2}$ target model was established, and then the Swerling target model in three cases was analyzed and the detection performance curve of the wide band target was deduced. Based on the radar equation, the influence of bandwidth on target detection performance is described in terms of incoherent accumulation efficiency (E), undulating loss (L) and target RCS, and the definition of frequency diversity of wideband radar is given. A Monte Carlo simulation experiment was designed to obtain the optimal number of resolution elements under different detection probabilities.

In this paper, on the basis of previous work, the gain performance of incoherent accumulation of wideband radar was studied systematically, a fair experimental environment was established to analyze diversity gain and the frequency diversity gain of wideband radar was defined. The optimal diversity gain corresponding to different target types under different detection probability requirements was given, that is, the number of optimal range units of different targets. For example, when the detection probability requirement is 0.9 , the Swerling II targets need to be resolved into 5-6 range units, and for a Swerling IV-type target, the target needs to be resolved into two range units.

In general, in the high-detection probability interval, a wideband signal is more advantageous; in other words, incoherent accumulation is more advantageous. However, this does not mean that the wider the signal bandwidth is better. Different targets are distinguished into a fixed number of range units, corresponding to different bandwidth, which means that the smaller target is more suitable for the wider signal, whereas the signal bandwidth required by the large target is much smaller. 
Author Contributions: All authors have made substantial contributions to this work. M.S. and F.H. performed the equations for the theoretical framework. M.S., F.H. and Z.D. designed the simulations; M.S. carried out the simulation experiments. M.S., F.H. and Z.D. analyzed the simulated data. M.S. wrote the manuscript. F.H., X.C., L.Y. and Z.D. reviewed and edited the manuscript. M.W. gave insightful and enlightening suggestions for this manuscript. All authors have read and agreed to the published version of the manuscript.

Funding: This research received no external funding.

Data Availability Statement: The data presented in this study are available on request from the corresponding author.

Acknowledgments: The authors would like to thank all those who gave valuable help and suggestions to this manuscript, especially my mentors and the editorial teachers at MDPI, who were essential to the outcome of this paper.

Conflicts of Interest: The authors declare no conflict of interest.

\section{Appendix A}

The flow chart of orthogonal demodulation is shown in Figure 1. Using trigonometric identity, the real and imaginary parts of the signal are, respectively, multiplied by the corresponding demodulation function; the results are as follows:

$$
\begin{aligned}
& s_{c 1}(\tau, n)=\frac{\sqrt{a_{n}}}{2} \cos \left(-2 \pi f_{0} t_{n}+\pi K_{r}\left(t-t_{n}\right)^{2}\right)+\frac{\sqrt{a_{n}}}{2} \cos \left(-2 \pi f_{0}\left(2 t-t_{n}\right)+\pi K_{r}\left(t-t_{n}\right)^{2}\right) \\
& s_{s 1}(\tau, n)=\frac{\sqrt{a_{n}}}{2} \sin \left(-2 \pi f_{0} t_{n}+\pi K_{r}\left(t-t_{n}\right)^{2}\right)+\frac{\sqrt{a_{n}}}{2} \sin \left(-2 \pi f_{0}\left(2 t-t_{n}\right)+\pi K_{r}\left(t-t_{n}\right)^{2}\right)
\end{aligned}
$$

The low-pass filter is used to process Equation (A1), and the results are as follows:

$$
\begin{aligned}
& s_{c 2}(\tau, n)=\frac{A_{n}^{\prime}}{2} \cos \left(-2 \pi f_{0} \tau_{n}+\pi K_{r}\left(\tau-\tau_{n}\right)^{2}\right) \\
& s_{s 2}(\tau, n)=\frac{\sqrt{a_{n}}}{2} \sin \left(-2 \pi f_{0} t_{n}+\pi K_{r}\left(t-t_{n}\right)^{2}\right)
\end{aligned}
$$

Due to the orthogonal relationship between the two signals, Equation (A2) can be expressed in the plural form as:

$$
\begin{aligned}
s_{r 2}(\tau, n) & =s_{c 2}(\tau, n)+j s_{s 2}(\tau, n) \\
& =\frac{\sqrt{a_{n}}}{2} \cos \left(-2 \pi f_{0} \tau_{n}+\pi K_{r}\left(\tau-\tau_{n}\right)^{2}\right)+\frac{\sqrt{a_{n}}}{2} j \sin \left(-2 \pi f_{0} \tau_{n}+\pi K_{r}\left(\tau-\tau_{n}\right)^{2}\right) \\
& =\frac{\sqrt{a_{n}}}{2} \exp \left(j \pi\left(-2 f_{0} t_{n}+K_{r}\left(t-t_{n}\right)^{2}\right)\right)
\end{aligned}
$$

The parameter definitions in the above Equations can be referred to the paper.

\section{Appendix B}

The basic expression of the Bessel function can be expressed as:

$$
I_{\alpha}(z)=\sum_{m=0}^{\infty} \frac{(-\mathbf{1})^{m}}{m ! \Gamma(m+\alpha+\mathbf{1})}\left(\frac{z}{\mathbf{2}}\right)^{2 m+\alpha}
$$

where, $\alpha$ is the order of the Bessel function, when $\alpha=1$, corresponding to the Bessel function of the first kind:

$$
I_{\mathbf{0}}(z)=\sum_{m=\mathbf{0}}^{\infty} \frac{(-\mathbf{1})^{m}}{m ! \Gamma(m+\mathbf{1})}\left(\frac{z}{\mathbf{2}}\right)^{2 m}
$$

The parameter definitions in the above Equations can be referred to in the paper. 


\section{References}

1. Wang, W.; An, D.; Luo, Y.; Zhou, Z. The Fundamental Trajectory Reconstruction Results of Ground Moving Target from Single-Channel CSAR Geometry. IEEE Trans. Geosci. Remote Sens. 2018, 56, 1-11. [CrossRef]

2. Zhang, L.; Xing, M.; Qiu, C.; Li, J.; Bao, Z. Achieving higher resolution ISAR imaging with limited pulses via compressive sampling. IEEE Geosci. Remote Sens. Lett. 2009, 6, 567-571. [CrossRef]

3. Kong, L.; Li, X.; Cui, G.; Yi, W.; Yang, Y. Coherent integration algorithm for a maneuvering target with high-order range migration. IEEE Trans. Signal Process. 2015, 63, 4474-4486. [CrossRef]

4. Yang, J.; Qiu, X.; Shang, M.; Zhong, L.; Ding, C. A Method of Marine Moving Targets Detection in Multi-Channel ScanSAR System. Remote Sens. 2020, 12, 3792. [CrossRef]

5. Bao, Z.; Xing, M.; Wang, T. Radar Imaging Technique; Publishing House of Electronics Industry: Beijing, China, 2005.

6. Kay, S. Waveform Design for Multistatic Radar Detection. IEEE Trans. Aerosp. Electron. Syst. 2009, 45, 1153-1166. [CrossRef]

7. O'Donnell, R.; Muehe, C. Automated Tracking for Aircraft Surveillance Radar Systems. IEEE Trans. 1979, 15, 508-517. [CrossRef]

8. Dai, F.; Liu, H.; Wu, S. Comparison of detection performance of wideband and narrowband radar in noise. J. Electron. Inf. 2010, 32, 1837-1842. [CrossRef]

9. Wehner, D.R. High Resolution Radar, 2nd ed.; Artech House Publishing: Boston, MA, USA; London, UK, 1994.

10. Orlenko, V.M.; Shirman, Y.D. Non-coherent intergration losses of wideband target detection. In Proceedings of the First European Radar Conference, Amsterdam, The Netherlands, 11-15 October 2004; pp. 225-228.

11. Xiao, J.; Huang, P.; Wen, S. Analysis of the influence of radar bandwidth on detection performance. In Proceedings of the 10th National Radar Annual Conference, Guangzhou, China, 6-8 May 2017; National Defense Industry Press: Beijing, China, 2017; pp. 39-43.

12. Jia, S. Research on Target Detection Algorithm of Wideband Radar; University of Electronic Science and Technology: Chengdu, China, 2010.

13. Li, Y. Research on Detection Method of Wideband Radar Target Echo Signal; University of Electronic Science and Technology: Chengdu, China, 2007.

14. Merrill, I. Introduction to Radar Systems, 3rd ed.; McGraw-Hall: New York, NY, USA, 2001.

15. Fran, C.; Nikita, P. Diversity considerations in wideband radar detection of migrating targets in clutter. Sci. China Inf. Sci. 2019, 62, 31-40.

16. William, L.; James, A. Principles of Modern Radar Advanced Techniques; SciTech Publishing: Edison, NJ, USA, 2001.

17. Tian, M.; Liao, G.; Zhu, S.; He, X.; Liu, Y.; Li, Y. An Efficient Method for Ground Maneuvering Target Refocusing and Motion Parameter Estimation Based on DPT-KT-MFP. Remote Sens. 2021, 13, 1092. [CrossRef]

18. Zhou, W.; Zhang, B.; Li, G. Maximum Likelihood Detector in Gamma-Distributed Sea Clutter. IEEE Geosci. Remote. Sens. Lett. 2018, 15, 1705-1709. [CrossRef]

19. Albersheim, W.J. A Closed-Form Approximation to Robertson's Detection Characteristics. Proc. IEEE 1981, 69, 839. [CrossRef]

20. Ding, L.; Geng, F.; Chen, J. Radar Principles; Northwestern Institute of Telecommunications Engineering Press: Xi'an, China, 1984.

21. Swerling, P. Probability of detection for fluctuating targets. IRE Trans. Inf. Theory 1960, 6, 269-308. [CrossRef]

22. Aittomaki, T.; Koivunen, V. Performance of MIMO radar with angular diversity under Swerling scattering models. IEEE J. Sel. Top. Signal Process. 2010, 4, 101-114. [CrossRef]

23. Zheng, J.; Zhu, K.; Niu, Z.; Liu, H.; Liu, Q.H. Generalized Dechirp-Keystone Transform for Radar High-Speed Maneuvering Target Detection and Localization. Remote Sens. 2021, 13, 3367. [CrossRef]

24. Zou, Y.; Tian, J.; Jin, G.; Zhang, Y. MTRC-Tolerated Multi-Target Imaging Based on 3D Hough Transform and Non-Equal Sampling Sparse Solution. Remote Sens. 2021, 13, 3817. [CrossRef] 